Pacific Journal of Mathematics

DIFFERENTIAL GEOMETRY OF SYSTEMS OF PROJECTIONS Gustavo CoRAch, Horacio Porte and Lázaro Rect 


\title{
DIFFERENTIAL GEOMETRY OF SYSTEMS OF PROJECTIONS IN BANACH ALGEBRAS
}

\author{
Gustavo Corach, Horacio Porta, and Lázaro Recht
}

Let $A$ be a Banach algebra, $n$ a positive integer and $Q_{n}=$ $\left\{\left(q_{1}, \ldots, q_{n}\right) \in A^{n}: q_{i} q_{k}=\delta_{l k} q_{i}, q_{1}+\cdots+q_{n}=1\right\}$. The differential geometry of $Q_{n}$, as a discrete union of homogeneous spaces of the group $G$ of units of $A$ is studied, a connection on the principal bundle $G \rightarrow Q_{n}$ is defined and invariants of the associated connection on the tangent bundle $T Q_{n}$ are determined.

Introduction. The structure of the set $Q$ of all idempotent elements of a Banach algebra $A$ plays a fundamental role in several aspects of spectral theory. This work deals with the differential structure of the space

$$
Q_{n}=\left\{\left(q_{1}, \ldots, q_{n}\right) \in A^{n}: q_{i} q_{k}=\delta_{i k} q_{i}, \sum_{i=1}^{n} q_{i}=1\right\}
$$

of systems of $n$ "orthogonal" projections in $A$.

The manifold $Q_{n}$ appears as a universal model when certain polynomial equations are considered. More precisely, if $\alpha_{1}, \ldots, \alpha_{n}$ are different complex numbers and $\alpha(X)$ denotes the polynomial $\left(X-\alpha_{1}\right) \cdots\left(X-\alpha_{n}\right)$, then the set $A_{\alpha}=\{a \in A: \alpha(a)=0\}$ is a closed submanifold which is diffeomorphic to $Q_{n}$. Thus $Q_{n}$ is the model for all simple algebraic elements of $A$ of degree $n$. Moreover, $Q_{n}$ plays a role in the study of arbitrary algebraic (in particular, nilpotent) elements (see [AS]).

Section 1 contains the description of the differential structure of $Q_{n}$ and $A_{\alpha}$ as closed analytic submanifolds of $A^{n}$ and $A$, respectively; it contains also the proof that $Q_{n}$ and $A_{\alpha}$ are diffeomorphic.

Using Kaplansky's notion of SBI-rings, we recover a result of Barnes [Ba] concerning the surjectivity of $A_{\alpha} \rightarrow B_{\alpha}$ when $B$ is the quotient of $A$ by its Jacobson radical. In $\S 2$ we show that $Q_{n}$ is a discrete union of homogeneous spaces of $G$, the group of units of $A$; this fact, together with a classical result of Michael [Mi], shows that an epimorphism $f: A \rightarrow B$ of Banach algebras induces Serre fibrations $Q_{n}(A) \rightarrow Q_{n}(B)$ and $A_{\alpha} \rightarrow B_{\alpha}$. In $\S 3$ we obtain an explicit way of 
lifting differentiable curves in $Q_{n}$ to $G$ by solving a linear differential equation which we call the transport equation; this fact is due to Daleckii and S. G. Krein [DK] and T. Kato [Ka1] but its geometrical meaning is new. In fact, in $\S 4$ we define a connection in the principal bundle $G \rightarrow Q_{n}$ and show that the horizontal liftings of differentiable curves in $Q_{n}$ are precisely the solutions of the transport equation.

Several invariants of the tangent bundle of $Q_{n}$ are calculated in $\S 5$ (covariant derivative, curvature, geodesics, etc.). As observed by Kato [Ka1], [Ka2, II.4] the lifting theorem has important applications in quantum mechanics (see [Ga], [GS]). A remark about $C^{*}$-algebras is in order: our results extend to the case of some involution algebras, in particular to all $C^{*}$-algebras. For instance, the transport equation has a unitary solution if the curve has selfadjoint values; in a forthcoming paper the immersion of

$$
P_{n}=\left\{p \in Q_{n}: p_{i}^{*}=p_{i}, i=1, \ldots, n\right\}
$$

into $Q_{n}$ will be studied, together with associated fibrations $Q_{n} \rightarrow P_{n}$.

Concerning the references, the reader may consult Rickart's book [Ri] for the literature up to 1960; the topology of the space of idempotents $Q=Q_{2}$ has been considered in [PR1], [Ra], [Ko], [Ze], [Au], [Gr] and with special emphasis on the differential struture of $Q$ in [Ra], [Gr], [Ki], [HK]; for the transport equation the reader may consult [Ka1] and [DK2]; in [PR2] the differential geometry of $P=P_{2}$ is needed for the study of minimality of geodesics; see also [CPR2] for a related problem; finally, the case of algebraic operators on Hilbert space, the reader may consult the books [He] and [AFVH]. In particular, some problems concerning the set $P_{n}$ in this context are discussed in [CH]. The set $Q_{n}$ appears, implicitly or explicitly, in various works; we only mention [Ja, p. 54], [Ka2, II.5] and [DK2, Chapter IV].

1. Differential structure of systems of projections. Let $A$ be a real or complex algebra with identity 1 . Denote by $G=G(A)$ the group of units of $A$ and by $Q=Q(A)$ the set of all idempotents of $A$.

Suppose that the polynomial $\alpha(X)=\prod_{i=1}^{n}\left(X-\alpha_{i}\right)$ has different roots $\alpha_{1}, \ldots, \alpha_{n}$ in the field. Let $g_{j}(X)=\prod_{i \neq j}\left(X-\alpha_{i}\right)$ and $q_{j}(X)=g_{j}(X) / g_{j}\left(\alpha_{j}\right)$. Then $q_{j}(X)$ has degree $n-1, q_{j}\left(\alpha_{i}\right)=\delta_{j i}$, for $i \neq j \quad q_{i}(X) q_{j}(X)=h(X) \alpha(X)$ for some polynomial $h(X)$ and $\sum_{i=1}^{n} q_{i}(X)=1$ (because $1-\sum_{i=1}^{n} q_{i}(X)$ has degree $\leq n-1$ and it vanishes at $n$ values, the $\alpha_{j}$ ).

Let $A_{\alpha}$ denote the solution set of $\alpha$, i.e., the set of all $a \in A$ with $\alpha(a)=0$. 
1.1. Proposition. Let $a \in A(\alpha)$. Then

(i) $\sum_{i=1}^{n} q_{i}(a)=1$;

(ii) $q_{i}(a) q_{j}(a)=0$ if $i \neq j$

(iii) $q_{i}(a) \in Q, i=1, \ldots, n$;

(iv) $q_{i}(a) a=a q_{i}(a)=\alpha_{i} q_{i}(a), i=1, \ldots, n$.

Proof. (i) follows from $\sum_{i=1}^{n} q_{i}(X)=1$ and (ii) follows from the equality $q_{i}(X) q_{j}(X)=h(X) \alpha(X)$. From (i) and (ii),

$$
q_{i}(a)=q_{i}(a) \sum_{k=1}^{n} q_{k}(a)=\sum_{k=1}^{n} q_{i}(a) q_{k}(a)=q_{i}(a)^{2},
$$

which gives (iii). Finally from $\alpha(X)=c\left(X-\alpha_{i}\right) q_{i}(X)$ (with $c=$ $\left.g_{i}\left(\alpha_{i}\right) \neq 0\right)$ it follows that $0=\alpha(a)=c\left(a q_{i}(a)-\alpha_{i} q_{i}(a)\right)$ and this completes the proof because $q_{i}(a)$ commutes with $a$.

Let $Q_{n}=Q_{n}(A)$ denote the set of all $n$-tuples of idempotents $q_{i}$ of $A$ which satisfy $q_{i} q_{j}=0$ if $i \neq j$ and $\sum_{i=1}^{n} q_{i}=1$.

1.2. Proposition. The mapping $a \rightarrow\left(q_{i}(a), \ldots, q_{n}(a)\right)$ is a bijection from $A_{\alpha}$ onto $Q_{n}$ whose inverse is $\left(q_{1}, \ldots, q_{n}\right) \rightarrow \sum_{i=1}^{n} \alpha_{i} q_{i}$.

The proof is a straightforward application of Proposition 1.1. Thus, from a set-theoretical view point, $Q_{n}$ is a universal model for the sets $A_{\alpha}$. We shall extend this result to the differential geometry setting.

1.3. REMARK. I. Kaplansky introduced the notion of SBI-rings (SBI = suitable for building idempotents) as those rings $A$ such that the natural mapping $Q(A) \rightarrow Q(A / R)$ is onto, where $R$ is the Jacobson radical of $A$.

It is known that for a SBI-ring $A$, the map $Q_{n}(A) \rightarrow Q_{n}(A / R)$ is also onto for each $n=1,2, \ldots$ (see [Ja, p. 54]).

It is also known that all Banach algebras are SBI [Ri, p. 58]. These facts and 1.2 imply that, for every $\alpha=\left(\alpha_{1}, \ldots, \alpha_{n}\right)$ (with $\alpha_{l} \neq \alpha_{k}$ ), $A_{\alpha} \rightarrow(A / R)_{\alpha}$ is onto, a result due to Barnes [Ba, Theorem 7].

From now on, we will assume that $A$ is a real or complex Banach algebra with identity. For $n$-tuples $Z=\left(Z_{1}, \ldots, Z_{n}\right)$ in $A^{n}$ we use the norm $\|Z\|=\max _{1 \leq i \leq n}\left\|Z_{i}\right\|$. The general facts on Banach algebras and Banach manifolds needed below can be found in [Ri] and [La], respectively. 
1.4. Theorem. Let $a \in A_{\alpha}$ be a fixed element, $q=q(a)=$ $\left(q_{1}(a), \ldots, q_{n}(a)\right) \in Q_{n}$ the corresponding system of idempotents. Set

$$
\begin{aligned}
& T=\left\{X \in A ; q_{i} X q_{i}=0 \text { for all } i=1, \ldots, n\right\}, \\
& S=\left\{Y \in A ; q_{k} Y q_{l}=0 \text { for all } k \neq l\right\} .
\end{aligned}
$$

1.4.(i) $A$ is the Banach space direct sum $A=T \oplus S$.

1.4.(ii) For each $Z=X+Y, X \in T, Y \in S$, set

$$
X^{\prime}=\sum_{i \neq k} q_{i} X q_{k} /\left(\alpha_{k}-\alpha_{i}\right)
$$

and define

$$
\phi(Z)=\exp \left(X^{\prime}\right)(a+Y) \exp \left(-X^{\prime}\right) .
$$

Then $\phi$ is a diffeomorphism from a neighborhood $U$ of $O \in A$ onto a neighborhood $V$ of $a$. Moreover, $\left.\phi\right|_{U \cap T}$ is a homeomorphism onto $V \cap A_{\alpha}$.

Proof. It is clear that every $Z \in A$ decomposes as $X+Y$, where

$$
\begin{aligned}
& X=\sum_{j \neq k} q_{j} Z q_{k} \in T \text { and } \\
& Y=\sum_{l} q_{l} Z q_{l} \in S, \quad \text { for } \sum_{l=1}^{n} q_{l}=1 \text { and } \\
& Z=\left(\sum q_{l}\right) Z\left(\sum q_{l}\right)=\sum_{j \neq k} q_{j} Z q_{k}+\sum_{l} q_{l} Z q_{l} .
\end{aligned}
$$

It is also clear that the decomposition is topological, for $T$ and $S$ are respectively defined as the images of the projections

$$
Z \rightarrow \sum_{j \neq k} q_{j} Z q_{k} \quad \text { and } \quad Z \rightarrow \sum_{l} q_{l} Z q_{l} .
$$

An easy computation shows that the derivative of $\phi$ at $O$ is the identity: in fact, for $Y \in S D \phi(O) Y=Y$ obviously; for $X \in T$ $D \phi(O) X=\left[X^{\prime}, a\right]=X^{\prime} a-a X^{\prime}=X$; the assertion follows from the decomposition $A=T \oplus S$.

Then, by the inverse function theorem, there exist open neighborhoods $U^{\prime}$ of $O$ and $V^{\prime}$ of $a$ such that $\phi$ maps $U^{\prime}$ diffeomorphically onto $V^{\prime}$. Consider next $Z=X+Y$ with $\phi(Z) \in A_{\alpha}$. Since 
$\phi(Z)=M(a+Y) M^{-1}$, then $a+Y$ is also a root of $\alpha$. Then $O=\prod_{i}\left(a+Y-\alpha_{i}\right)$ and using Prop. 1.1.(iv):

$$
\begin{aligned}
O & =q_{j} \prod_{i}\left(a+Y-\alpha_{i}\right)=q_{j} \prod_{i}\left(\alpha_{j}+Y-\alpha_{i}\right) \\
& =q_{j} Y L
\end{aligned}
$$

where $L=\prod_{j \neq i}\left(Y-\left(\alpha_{i}-\alpha_{j}\right)\right)$. If $Y$ has small norm $(\|Y\|<$ $\min \left\{\left|\alpha_{i}-\alpha_{j}\right|, i \neq j\right\}$ suffices) then $L$ is invertible and therefore $q_{j} Y=0$ for each $j$. Hence $\phi(Z) \in A_{\alpha}$ with $Y$ small implies $Z \in T$. This means that (perhaps for smaller neighborhoods) $\phi$ is a homeomorphism from $U^{\prime} \cap T$ onto $V^{\prime} \cap V_{\alpha}$.

Considering the maps $\phi$ as analytic local coordinates in $A$, we obtain:

1.5. Corollary. $A_{\alpha}$ is a closed analytic submanifold of $A$ whose tangent space at $a \in A_{\alpha}$ can be identified to the Banach space $T$.

1.6. REMARKs. (i) The choice of the chart $\phi$ may seem rather artificial; for instance, the derivative at $O$ of $\phi_{1}(X+Y)=$ $\exp (X)(a+Y) \exp (-X)$ is $X+Y \rightarrow X a-a X+Y=[X, a]+Y$ and the equalities $q_{i}[X, a] q_{j}=\left(\alpha_{j}-\alpha_{i}\right) q_{i} X q_{j} \quad(i \neq j)$ show that $D \phi_{1}(O)$ maps $T$ onto $T$ and $S$ onto $S$. Thus, $\phi_{1}$ also provides charts for the analytic structure of $A_{\alpha}$. However, we have chosen the map $\phi$ because it is the exponential map of the natural connection to be studied later (see $\S 4$ ). This remarks applies also to the charts chosen below for $Q_{n}$.

(ii) An obvious consequence of 1.3 is that $A_{\alpha}$ is locally arcwise connected for all $\alpha$ as above. For the simpler case of $\alpha(X)=X(X-1)$ this is a result of Zemanek [Ze, 3.2] for complex Banach algebras, which was generalized for real algebras by Aupetit [Au, p. 413]. However both results have been also proved in [PR1, 4.3] (see also 2.2(iii) below).

1.7. TheOREM. $Q_{n}$ is a closed submanifold of $A^{n}$.

Proof. Fix $q \in Q_{n}$ and define $T^{\prime}=\left\{X=\left(X_{1}, \ldots, X_{n}\right) \in A^{n}: q_{r} X_{i} q_{s}\right.$ $=0$ for $r \neq i$ and $s \neq i$ or $r=s=i$, and $q_{i} X_{i} q_{k}+q_{i} X_{k} q_{k}=0$ for $i \neq k\}$. 
The map $\theta: A^{n} \rightarrow A^{n}, \quad \theta\left(Z_{i}, \ldots, Z_{n}\right)=\left(X_{1}, \ldots, X_{n}\right)$ defined by

$$
\begin{aligned}
X_{1} & =\sum_{i>1} q_{1} Z_{1} q_{i}+q_{i} Z_{1} q_{1}, \\
X_{2} & =\left(\sum_{i>2} q_{2} Z_{2} q_{i}+q_{i} Z_{2} q_{i}\right)-\left(q_{1} Z_{1} q_{2}+q_{2} Z_{1} q_{1}\right), \\
\vdots & \\
X_{k} & =\sum_{i>k}\left(q_{k} Z_{k} q_{i}+q_{i} Z_{k} q_{k}\right)-\sum_{i<k}\left(q_{i} Z_{i} q_{k}+q_{k} Z_{i} q_{i}\right) \quad(k \leq n-1), \\
X_{n} & =-\sum_{k=1}^{n-1} X_{k}
\end{aligned}
$$

is a projection onto $T^{\prime}$ whose kernel is the set $S^{\prime}$ of all $Y=\left(Y_{1}, \ldots\right.$, $\left.Y_{n}\right) \in A^{n}$ with $q_{r} Y_{i} q_{s}=0$ for $r=i$ and $s>i$ or $s=i$ and $r>i$.

Thus $T^{\prime} \oplus S^{\prime}=A^{n}$. For $X \in T^{\prime}$ put

$$
\tilde{X}=\sum_{i \neq j} \widetilde{X}_{i j} \quad \text { where } \widetilde{X}_{i j}= \begin{cases}q_{i} X_{j} q_{j} & \text { if } j<i, \\ -q_{i} X_{i} q_{j} & \text { if } i<j .\end{cases}
$$

Observe that $q_{i} \tilde{X} q_{i}=0$ for $i=1, \ldots, n$.

Consider now the map $\psi: A^{n} \rightarrow A^{n}$ defined by

$$
\psi(Z)_{i}=\psi(X+Y)_{i}=\exp (\tilde{X})\left(q_{i} Y_{i}\right) \exp (-\tilde{X})
$$

for $X \in T^{\prime}, Y \in S^{\prime}$. Then $D \psi(O) Y=Y$ for $Y \in S^{\prime}$ and, calculating,

$$
(D \psi(O) X)_{i}=\left[\tilde{X}, q_{i}\right]=X_{i} \text { for } X \in T^{\prime}, i=1, \ldots, n .
$$

This means that $D \psi(O)=$ identity and $\psi$ is a diffeomorphism from a neighborhood of $O$ onto a neighborhood of $q$. For $Y \in S^{\prime}$ such that $\|Y\|<1$ it is easily shown that $q+Y \in Q_{n}$ if and only if $Y=O$. This completes the proof.

Remark. According to Proposition 1.2, the bijections connecting $A_{\alpha}$ and $Q_{n}$ are given by algebraic expressions.

The next result, whose proof follows easily from the theorems above, shows that $Q_{n}$ is a universal model for the sets $A_{\alpha}$ of simple algebraic elements of degree $n$.

1.8. Theorem. The map $a \rightarrow\left(q_{1}(a), \ldots, q_{n}(a)\right)$ is a diffeomorphism from $A_{\alpha}$ onto $Q_{n}$ whose inverse is given by $\left(q_{1}, \ldots, q_{n}\right) \rightarrow$ 
$\sum_{i=1}^{n} \alpha_{i} q_{i}$. Consequently, for any other $\beta=\left(\beta_{1}, \ldots, \beta_{n}\right)$ with $\beta_{i} \neq$ $\beta_{j}$ the map $a \rightarrow \sum_{i=1}^{n} \beta_{i} q_{i}(a)$ is a diffeomorphism from $A_{\alpha}$ onto $A_{\beta}$.

2. Fibrations. The group $G$ of invertible elements of $A$ acts on $Q_{n}$ by inner automorphisms on each coordinate: if $g \in G$ and $q=$ $\left(q_{1}, \ldots, q_{n}\right) \in P_{n}$ then $g q g^{-1}=\left(g q_{1} g^{-1}, \ldots, g q_{n} g^{-1}\right) \in Q_{n}$.

2.1. THEOREM. Let $q$ be a fixed element of $Q_{n}$ and define $\pi: G \rightarrow$ $Q_{n}$ by $\pi(g)=g q g^{-1}$. Then

(i) there exist an open neighborhood $U$ of $q$ in $Q_{n}$ and a local section $\sigma: U \rightarrow G$ of $\pi$;

(ii) the orbit $V_{q}=\left\{g q g^{-1}: g \in G\right\}$ is open (and closed) in $Q_{n}$;

(iii) $\pi: G \rightarrow V_{q}$ is a principal fiber bundle with structure group $G_{0}=$ $\left\{g \in G: g q_{1}=q_{1} g, i=1, \ldots, n\right\}$.

Therefore $Q_{n}$ is a discrete union of homogeneous spaces of $G$.

Proof. Given $q^{\prime} \in Q_{n}$ define

$$
\sigma\left(q^{\prime}\right)=\left\langle q, q^{\prime}\right\rangle=q_{1}^{\prime} q_{1}+\cdots+q_{n}^{\prime} q_{n} .
$$

It is clear that $\sigma(q)=1$ and $\sigma(q) q_{i}=q_{i}^{\prime} \sigma\left(q^{\prime}\right)$. Thus, for every $q^{\prime}$ in a neighborhood $U$ of $q$, we have $\sigma\left(q^{\prime}\right) \in G$ and $\sigma\left(q^{\prime}\right) q \sigma\left(q^{\prime}\right)^{-1}=q^{\prime}$. This proves (i) and (ii) and the rest of the statement follows from standard arguments (see [St, §7]).

2.2. Remarks. (i) An invertible element $g$ belongs to $G_{0}$ if and only if $q_{k} g q_{l}=0$ for all $k \neq l$. Thus, the Lie algebra of $G_{0}$ can be identified to $\left\{X \in A: q_{k} X q_{l}=0\right.$ for all $\left.k \neq l\right\}$.

(ii) With the notations of 2.1 and 1.6 it is easy to describe trivializations of the tangent bundle $T Q_{n}$ and of a suplement $N Q_{n}$ of $T Q_{n}$ in the trivial bundle $\varepsilon: Q_{n} \times A^{n} \rightarrow Q_{n}$. We call $N Q_{n}$ the "normal bundle" of $Q_{n}$. Given $q \in Q_{n}$, let $U_{q}=\left\{q^{\prime} \in Q_{n}: \sigma\left(q^{\prime}\right) \in G\right\}$. Then $h: U_{q} \times A^{n} \rightarrow U_{q} \times A^{n}$, defined by

$$
h\left(q^{\prime}, Z\right)=\left(q^{\prime}, \sigma\left(q^{\prime}\right) Z \sigma\left(q^{\prime}\right)^{-1}\right),
$$

is a diffeomorphism which trivializes simultaneously $\tau: T Q_{n} \rightarrow Q_{n}$ and a bundle $\nu: N Q_{n} \rightarrow Q_{n}$ where $\left(N Q_{n}\right)_{q}=S^{\prime}$ (as in 1.6).

(iii) Given $q \in Q_{n}$, its connected component (in $Q_{n}$ ) can be described as the set $\left\{g q g^{-1}: g \in G^{0}\right\}$, where $G^{0}$ is the connected component of 1 in $G$ : in fact, it suffices to replace $G$ by $G^{0}$ in the proof of 2.1. Of course, similar statements hold for $A_{\alpha}$. This generalizes [Ze, Theorem 3.3] and [Au]. 
2.3. Corollary. Consider a fixed $q \in Q_{n}$ and a continuous curve $\gamma:[0,1] \rightarrow Q_{n}$ such that $\gamma(0)=q$. Then, there exists a continuous curve $\Gamma:[0,1] \rightarrow G$ such that $\Gamma(0)=1$ and $\pi \circ \gamma=\gamma$, where $\pi(g)=$ $g q g^{-1}$.

We consider now the behaviour of the functor $Q_{n}$ under epimorphisms.

Let $f: A \rightarrow B$ be a continuous homomorphism of Banach algebras which preserves the identity

Clearly $f$ induces maps $G(f): G(A) \rightarrow G(B)$, and $f_{n}: Q_{n}(A) \rightarrow$ $Q_{n}(B)$. We shall prove that $f_{n}$ is a Serre fibration when $f$ is an epimorphism [Sp].

2.4. TheOREM. Let $f: A \rightarrow B$ be a (continuous) epimorphism of Banach algebras. Then $f_{n}: Q_{n}(A) \rightarrow Q_{n}(B)$ is a Serre fibration. In particular, $f_{n}$ is onto if and only if its image intersects every connected component of $Q_{n}(B)$.

Proof. Replacing $A$ and $B$ by $C\left(I^{m}, A\right)$ (= algebra of all maps $\left.I^{m} \rightarrow A\right)$ and $C\left(I^{m}, B\right)$ respectively (where $I=[0,1]$ ), it suffices to show that if $\gamma: I \rightarrow Q_{n}(B)$ is such that $\gamma(0)=q^{\prime}=f_{n}(q)$ for some $q \in Q_{n}(A)$ there exists a curve $\tilde{\gamma}: I \rightarrow Q_{n}(A)$ such that $f_{n} \circ \tilde{\gamma}=\gamma$.

For this, we consider the commutative diagram

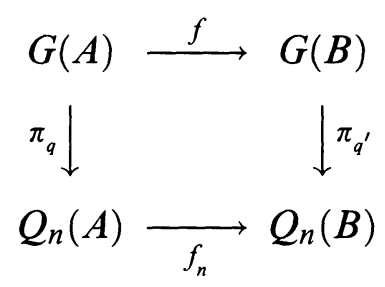

where $\pi_{q}(g)=g q g^{-1}, \pi_{q^{\prime}}(h)=h q^{\prime} h^{-1}(g \in G(A), h \in G(B))$. By the local triviality of $\pi_{q^{\prime}}$ proved in 2.1 , there is a curve $\delta: I \rightarrow G(B)$ with $\delta(0)=1$ and $\pi_{q^{\prime}} \delta=\gamma$. Michael [Mi] proved that $f: G(A) \rightarrow$ $G(B)$ is a Serre fibration; therefore, there is a curve $\varepsilon: I \rightarrow G(A)$ such that $\varepsilon(0)=1$ and $f \circ \varepsilon=\delta$. To finish the proof it suffices to define $\tilde{\gamma}=\pi_{q} \circ \varepsilon$, which satisfies $f_{n} \circ \tilde{\gamma}=\gamma$.

The next theorem extends results of Raeburn [Ra] concerning the set $\pi_{0}(P(A \hat{\otimes} B))$ of all connected components of the idempotents of $A \hat{\otimes} B$, where $A$ is supposed to be commutative.

We omit its proof and that of the proposition below because they are simple combination of Raeburn's techniques without previous results. 
2.5. Proposition (cf. [Ra, p. 383]). Let $A$ be a Banach algebra and $B_{1}, \ldots, B_{n}$ be open balls in $\mathbf{C}$ with pairwise disjoint closures, centered at $\alpha_{1}, \ldots, \alpha_{n}$, respectively. Let $U=B_{1} \cup \cdots \cup B_{n}$ and $A_{U}=\{a \in A$ : the spectrum of $a$ is contained in $U\}$. Then $A_{U}$ is open in $A$ and $f=\left(f_{1}, \ldots, f_{n}\right): A_{U} \rightarrow A^{n}$ is an analytic retraction onto $Q_{n}$, where $f_{i}: U \rightarrow \mathbf{C}$ is defined by $f_{i}(z)=\delta_{i k}$ for $z \in B_{k}$ and $f_{n}(a)$ is obtained by means of the holomorphic functional calculus.

2.6. Theorem (cf. [Ra, 4.5, 4.7]). Let $A$ and $B$ be complex Banach algebras. Suppose that $A$ is commutative with spectrum $X$. Then the Gelfand map $A \rightarrow C(X)$ induces bijections

$$
\begin{aligned}
\pi_{0}\left(Q_{n}(A \hat{\otimes} B)\right) & \rightarrow\left[X, Q_{n}(B)\right], \\
\left\{Q_{n}(A \hat{\otimes} B)\right\} & \rightarrow\left\{Q_{n}(C(X, B))\right\}
\end{aligned}
$$

where [, ] denotes homotopy classes of maps and $\left\{Q_{n}(C)\right\}$ is the set of orbits of the action of $G(C)$ on $Q_{n}(C)$.

2.7. REMARK. If $A$ is the algebra of complex continuous functions on the 3-sphere, $B$ is the algebra of all $2 \times 2$-matrices over $C$ and $n=2$, we reobtain the example of [PR1, 7.13].

3. Lifting $C^{1}$-curves. The transport equation. In this section we describe a method which leads to a lifting $\Gamma$ of a curve $\gamma:[a, b] \rightarrow$ $Q_{n}$, as in Corollary 2.3, valid when $\gamma$ is rectifiable and continuous. For the sake of simplicity we only consider $n=2$, the general case being similar and somewhat more involved. The reader can find the details (for $n=2$ ) in [PR1]. Our present interest in this construction lies in that it leads to the transport equation.

Consider a continuous rectifiable curve $\gamma:[a, t] \rightarrow Q$ and a partition $\Pi: t_{0}=a<t_{1}<\cdots<t_{n}=t$ such that $\left\|\gamma_{k}-\gamma_{k+1}\right\|<1$ $(k=0, \ldots, n-1)$, where $\gamma_{k}=\gamma\left(t_{k}\right)$; then

$$
\begin{gathered}
\sigma_{k}=\gamma_{k} \gamma_{k-1}+\left(1-\gamma_{k}\right)\left(1-\gamma_{k-1}\right) \in G \quad(k=0, \ldots, n-1) \quad \text { and } \\
\sigma_{k} \gamma_{0} \sigma_{1}^{-1}=\gamma_{1}, \\
\sigma_{2} \sigma_{1} \gamma_{0} \sigma_{1}^{-1} \sigma_{2}^{-1}=\sigma_{2} \gamma_{1} \sigma_{2}^{-1}=\gamma_{2}, \ldots, \sigma_{n} \cdots \sigma_{1} \gamma_{0} \sigma_{1}^{-1} \cdots \sigma_{n}^{-1}=\gamma_{n} .
\end{gathered}
$$

Thus, $\sigma$ can be thought of as a "discrete" curve of units which conjugates $\gamma_{0}$ with $\gamma_{n}$. Putting $u(\Pi)=\sigma_{n} \cdots \sigma_{1}$, it can be shown [PR1, $\S 5]$ that the limit $\Gamma(t)=\lim u(\Pi)$, when the length of the partition $\Pi$ tends to zero, exists and defines a unit of the algebra. Moreover 
$\Gamma:[a, b] \rightarrow G$ is continuous and rectifiable. If the original curve $\gamma$ has a continuous derivative, then the value

$$
\begin{aligned}
& (1 / h)(\Gamma(t+h)-\Gamma(t) \quad \text { is, approximately, } \\
& (1 / h)\left(\sigma_{t+h} \Gamma(t)-\Gamma(t)\right), \quad \text { where } \\
& \sigma_{t+h}=\gamma(t+h) \gamma(t)+(1-\gamma(t+h))(1-\gamma(t))
\end{aligned}
$$

Then,

$$
\begin{aligned}
(1 / h) & (\Gamma(t+h)-\gamma(t)) \cong(1 / h)\left(\sigma_{t+h}-1\right) \Gamma(t) \\
\quad & =(1 / h)(2 \gamma(t+h) \gamma(t)-\gamma(t+h)-\gamma(t)) \Gamma(t) \\
& =(1 / h)\{\gamma(t+h)(\gamma(t)-\gamma(t+h))+(\gamma(t+h)-\gamma(t)) \gamma(t)\} \Gamma(t)
\end{aligned}
$$

and

$$
\begin{aligned}
\dot{\Gamma}(t) & =\lim _{h \rightarrow 0}(1 / h)(\Gamma(t+h)-\Gamma(t)) \\
& =\{-\gamma(t) \dot{\gamma}(t)+\dot{\gamma}(t) \gamma(t)\} \Gamma(t) .
\end{aligned}
$$

Thus, the lifting $\Gamma$ of $\gamma$ constructed by the limiting process described above satisfies the initial values problem

$$
\begin{aligned}
& \dot{\Gamma}=(\dot{\gamma} \gamma-\gamma \dot{\gamma}), \\
& \Gamma(0)=1 .
\end{aligned}
$$

In the general case $n>2$ the initial value problem is

$$
\begin{aligned}
& \dot{\Gamma}=\left(\sum_{1}^{n} \dot{\gamma}_{k} \gamma_{k}\right) \Gamma, \\
& \Gamma(0)=1,
\end{aligned}
$$

where $\gamma=\left(\gamma_{1}, \ldots, \gamma_{n}\right):[a, b] \rightarrow Q_{n}$ is of class $C^{1}$. Observe that $\sum_{1}^{2} \dot{\gamma}_{k} \gamma_{k}=\dot{\gamma}_{1} \gamma_{1}-\dot{\gamma}_{1}\left(1-\gamma_{1}\right)=\dot{\gamma}_{1} \gamma_{1}-\gamma_{1} \dot{\gamma}_{1}$ because $\gamma_{2}=1-\gamma_{2}$ and $\dot{\gamma}_{1}=\dot{\gamma}_{1} \gamma_{1}+\gamma_{1} \dot{\gamma}_{1}$ (differentiate $\left.\gamma_{1}^{2}=\gamma_{1}\right)$.

As we said before, we shall not justify all the assertions about $\Gamma$. Instead we include the proof of the following result due to Daleckii, Krein and Kato, for the sake of completeness (see [DK2, IV, Theorem 1.1]).

3.1. Theorem. Let $\gamma:[a, b] \rightarrow Q_{n}$ be a $C^{1}$ curve. Then, the unique solution in $A$ of the initial conditions problem

$$
\begin{aligned}
& \dot{\Gamma}=\hat{\gamma} \Gamma, \\
& \Gamma(a)=1,
\end{aligned}
$$


where $\hat{\gamma}=\sum_{k=1}^{n} \dot{\gamma}_{k} \gamma_{k}$, satisfies

(i) $\Gamma(t) \in G(t \in[a, b])$,

(ii) $\Gamma(t) \gamma(a) \Gamma(t)^{-1}=\gamma(t) \quad(t \in[a, b])$.

Proof. Existence and uniqueness of $\Gamma$ follow from general facts [La, p. 71]. To prove (i) consider the companion problem

$$
\left\{\begin{array}{l}
\dot{\Delta}=-\Delta \hat{\gamma}, \\
\Delta(a)=1,
\end{array}\right.
$$

and observe that $(\Delta \Gamma)^{\cdot}=\dot{\Delta} \Gamma+\Delta \dot{\Gamma}=0$. Then $\Delta \Gamma$ is constant on $[a, b]$ and, since $\Delta(a)=\Gamma(a)=1$, it is $\Delta \Gamma \equiv 1$. Thus $\Gamma(t)$ is left invertible in $A$; moreover, $\Gamma(t)$ belongs to the connected component of the identity in the set of left invertible elements. It is easy to see that this component is completely contained in $G$. This proves (i).

To see (ii) we compute $\left(\Gamma^{-1} \gamma_{k} \Gamma\right)^{\cdot}(k=1, \ldots, n)$ :

$$
\begin{aligned}
\left(\Gamma^{-1} \gamma_{k} \Gamma\right)^{\cdot} & =-\Gamma^{-1} \dot{\Gamma} \Gamma^{-1} \gamma_{k} \Gamma+\Gamma^{-1} \dot{\gamma}_{k} \Gamma+\Gamma^{-1} \gamma_{k} \dot{\Gamma} \\
& =-\Gamma^{-1}\left\{\hat{\gamma} \gamma_{k}-\dot{\gamma}_{k}-\gamma_{k} \hat{\gamma}\right\} \Gamma
\end{aligned}
$$

observe that $\hat{\gamma} \gamma_{k}=\left(\sum \dot{\gamma}_{i} \gamma_{i}\right) \gamma_{k}=\dot{\gamma}_{k} \gamma_{k}$, because $\gamma_{i} \gamma_{k}=0$ for $i \neq k$, and that $\gamma_{k} \hat{\gamma}=\gamma_{k}\left(\sum \dot{\gamma}_{i} \gamma_{i}\right)=-\gamma_{k}\left(\sum \gamma_{i} \dot{\gamma}_{i}\right)=-\gamma_{k} \dot{\gamma}_{k}$, because $\dot{\gamma}_{k}=$ $\dot{\gamma}_{k} \gamma_{k}+\gamma_{k} \dot{\gamma}_{k}$ and $\sum \dot{\gamma}_{k}=\left(\sum \dot{\gamma}_{k}\right)^{\cdot}=1^{\cdot}=0$. Thus

$$
\left(\gamma^{-1} \gamma_{k} \Gamma\right)^{\cdot}=-\Gamma^{-1}\left\{\dot{\gamma}_{k} \gamma_{k}-\dot{\gamma}_{k}+\gamma_{k} \dot{\gamma}_{k}\right\} \Gamma=0
$$

and $\Gamma^{-1} \gamma_{k} \Gamma$ is constantly $\gamma_{k}(a)$. This completes the proof of (ii).

3.2. REMARK. The proof of part (i) could have been omitted because it is a general fact that the solution of $\dot{\Gamma}=\varphi \Gamma, \Gamma(a)=1$, where $\varphi:[a, b] \rightarrow A$ is a continuous curve, is a curve of invertible element of $A$.

If $A$ is an involutive Banach algebra, i.e. there exists a continuous antilinear mapping $x \rightarrow x^{*}$ such that $(x y)^{*}=y^{*} x^{*}, 1^{*}=1$ and $x^{* *}=x \quad(x, y \in A)$, we consider the unitary group of $A$

$$
U=\left\{u \in G: u^{-1}=u^{*}\right\}
$$

and the selfadjoint part of $Q_{n}$

$$
P_{n}=\left\{p=\left(p_{1}, \ldots, p_{n}\right) \in Q_{n}: p_{k}^{*}=p_{k} \quad(k=1, \ldots, n)\right\} .
$$

For these algebras more specific results hold. We omit the details about the differential structure of $P_{n}$. 
3.3. Corollary. If $\gamma:[a, b] \rightarrow P_{n}$ is a $C^{1}$ curve then the solution of $\dot{\Gamma}=\hat{\gamma} \Gamma, \Gamma(a)=1$, defines a curve $\Gamma:[a, b] \rightarrow U$ which conjugates the curve $\gamma$.

Proof. It suffices to show that $\Gamma(t) \in U$ for every $t \in[a, b]$. Observe first that

$$
\begin{aligned}
\dot{\Gamma}^{*} & =\left\{\left(\sum \dot{\gamma}_{k} \gamma_{k}\right) \Gamma^{*}=\Gamma^{*}\left(\sum \dot{\gamma}_{k} \gamma_{k}\right)^{*}\right. \\
& =\Gamma^{*}\left(\sum \gamma_{k} \dot{\gamma}_{k}\right)=-\Gamma^{*}\left(\sum \dot{\gamma}_{k} \gamma_{k}\right),
\end{aligned}
$$

because

$$
\sum \dot{\gamma}_{k} \gamma_{k}+\gamma_{k} \dot{\gamma}_{k}=\sum \dot{\gamma}_{k}=\left(\sum \gamma_{k}\right)^{\cdot}=1^{\cdot}=0 \text {. }
$$

Thus $\left(\Gamma^{*} \Gamma\right)^{\cdot}=\dot{\Gamma}^{*} \Gamma+\Gamma^{*} \dot{\Gamma}=0$ and $\Gamma^{*} \Gamma$ is constant. But $\Gamma(0)=$ $\Gamma^{*}(0)=1$, so $\Gamma^{*} \Gamma=1$. Now, $\Gamma(t)$ is invertible for all $t$, by Theorem 3.1, so $\Gamma(t)^{*}=\Gamma(t)^{-1}$.

3.4. REMARK. Of course many liftings of $\gamma$ may exist. But $\Gamma$ is the unique horizontal lifting of $\gamma$ with respect to the connection we shall define in the next section. This fact completes Kato's remark [Ka, II.4.2, Remark 4.4]. Moreover, if our $\sigma$ 's, used to obtain the transport equation, are multiplied (at left or at right) by $\left(1-\left(\gamma_{k}-\gamma_{k-1}\right)^{2}\right)^{-1 / 2}$, where $(1-r)^{-1 / 2}=\sum_{m=0}^{\infty}\left(\begin{array}{c}-1 / 2 \\ m\end{array}\right)(-r)^{m}$ for $\|r\|<1$, we get a different "discrete" lifting of $\gamma$ but in the limit it becomes the same continuous curve $\Gamma$. In this sense, the local solution [Ka, p. 102, (4.18)]

$$
\Gamma_{1}(t)=\left(1-(\gamma(t)-\gamma(0))^{2}\right)^{-1 / 2}(\gamma(t) \gamma(0)+(1-\gamma(t)))(1-\gamma(0))
$$

is related to the global solution $\Gamma$.

4. The connection. Let $q \in Q_{n}$ be fixed and $\pi: G \rightarrow Q_{n}$ defined by $\pi(g)=g q g^{-1}=\left(g q_{1} g^{-1}, \ldots, g q_{n} g^{-1}\right)$. It is very easy to show that the derivative of $\pi$ at $g \in G(T \pi)_{g}:(T G)_{g}:(T G)_{g} \rightarrow\left(T Q_{n}\right)_{\pi(g)}$ is given by

$$
(T \pi)_{g}(X)=g\left[g^{-1} X, q\right] g^{-1} \quad\left(X \in(T G)_{g}\right)
$$

where $[Z, q]=\left(\left[Z, q_{1}\right], \ldots,\left[Z, q_{n}\right]\right)$ for all $Z \in A$.

We say that $X \in(T G)_{g}$ is vertical if $(T \pi)_{g}(X)=0$ or, what is the same, if $\left[g^{-1} X, q\right]=0$. Then, if $V_{g}=\left\{X \in(T G)_{g}:\left[g^{-1} X, q\right]=0\right\}$, 
it is clear that $V_{g}=g \cdot V_{1}$ and that

$$
\begin{aligned}
V_{1} & =\left\{X \in A=(T G)_{g}:[X, q]=0\right\} \\
& =\left\{X \in A: q_{k} X q_{i}=0 \text { for all } i \neq k\right\} \\
& =\left\{\sum_{i=1}^{n} q_{i} X q_{i}: X \in A\right\} .
\end{aligned}
$$

This shows that

$$
\begin{aligned}
H_{1} & =\left\{X \in A: q_{i} X q_{i}=0(i=1, \ldots, n)\right\} \\
& =\left\{\sum_{k \neq i} q_{k} X q_{i}: X \in A\right\}
\end{aligned}
$$

is a supplement of $V_{1}$ in $A\left(=(T G)_{1}\right)$ and, in general $H_{g}=g H_{1}$ is a supplement of $V_{g}$ in $A\left(=(T G)_{g}\right)$. Moreover, $H_{g} \cdot h=H_{g h}(g \in G$, $h \in H)$. Finally, the projections $h_{g}:(T G)_{g} \rightarrow H_{g}, v_{g}:(T G)_{g} \rightarrow V_{g}$ given by

$$
\begin{aligned}
& h_{g}(X)=g \sum_{i \neq k} q_{k} g^{-1} X q_{i}, \\
& v_{g}(X)=g \sum_{i=1}^{n} q_{i} g^{-1} X q_{i},
\end{aligned}
$$

verify

$$
\begin{aligned}
& h_{g}(X)=g h_{1}\left(g^{-1} X\right), \\
& v_{g}(X)=g v_{1}\left(g^{-1} X\right) .
\end{aligned}
$$

Clearly the mappings $g \rightarrow h_{g}$ and $g \rightarrow v_{g}$ from $G$ into the bounded linear operators on $A$ are differentiable. All these facts show that $g \rightarrow H_{g}$ defines a connection in the principal bundle $\pi: G \rightarrow Q_{n}^{\prime}$.

For the theory of connections we refer the reader to [KN]. However, we are dealing with Banach manifolds and bundles, which requires a few notational changes.

From now on by "curve" we mean a $C^{\infty}$ curve.

Given a curve $\gamma:[\alpha, \beta] \rightarrow Q_{n}$, a horizontal lifting of $\gamma$ is a curve $\Gamma:[\alpha, \beta] \rightarrow G$ such that $\pi \Gamma=\gamma$ and $\dot{\Gamma}(t) \in H_{\Gamma(t)}(t \in[\alpha, \beta])$.

It is a general fact that, for each $g_{0} \in G$ such that $\gamma(\alpha)=g_{0} \mathrm{pg}_{0}^{-1}$, there is a unique horizontal lifting $\Gamma$ such that $\Gamma(\alpha)=g_{0}$. In particular, if $\gamma(\alpha)=q$ there is a unique horizontal lifting $\Gamma$ such that $\Gamma(\alpha)=1$. 
4.1. Theorem. Given a curve $\gamma:[\alpha, \beta] \rightarrow Q_{n}$ the horizontal lifting $\Gamma$ such that $\Gamma(\alpha)=1$ is the solution of the transport equation

$$
\dot{\Gamma}=\hat{\gamma} \Gamma, \quad \text { where } \hat{\gamma}=\sum_{i=1}^{n} \dot{\gamma}_{i} \gamma_{i},
$$

with initial condition $\Gamma(\alpha)=1$.

Proof. We have seen that the solution $\Gamma$ of (4.2) is a lifting of $\pi$, i.e. $\pi \circ \Gamma=\gamma$ (see 3.1). By the uniqueness of both objects it suffices to show that the horizontal lifting $\Gamma$ with $\Gamma(\alpha)=1$ satisfies (4.2). We recall that $\Gamma$ satisfies

$$
\Gamma(t) q \Gamma(t)^{-1}=\gamma(t) \quad(t \in[\alpha, \beta]),
$$

$$
\dot{\Gamma} \in H_{\Gamma}=\Gamma H_{1} \text {, i.e. } \dot{\Gamma}(t) \in \Gamma(t) H_{1} \quad(t \in[\alpha, \beta])
$$

or, what is the same

$$
\Gamma^{-1} \gamma \Gamma=q
$$

and

$$
\Gamma^{-1} \dot{\Gamma} \in H_{1}
$$

Differentiating (4.5) we get $0=\Gamma^{-1}\left(-\dot{\Gamma} \Gamma^{-1} \gamma+\dot{\gamma}+\gamma \dot{\Gamma} \Gamma^{-1}\right) \Gamma$ and cancelling $\Gamma^{-1}$ and $\Gamma$, we get

$$
\dot{\gamma}=\left[\dot{\Gamma} \Gamma^{-1}, \gamma\right] \text {. }
$$

Now, (4.6) means that $q_{i} \Gamma^{-1} \dot{\Gamma} q_{1}=0,(i=1, \ldots, n)$, which can also be written as

$$
q \Gamma^{-1} \dot{\Gamma}=\Gamma^{-1} \dot{\Gamma}(1-q)
$$

Replacing (4.5) in (4.8) we get $\Gamma^{-1} \gamma \dot{\Gamma}=\Gamma^{-1} \dot{\Gamma}-\Gamma^{-1} \dot{\Gamma} \Gamma^{-1} \gamma \Gamma$ which, after cancellation, gives

$$
\gamma \dot{\Gamma} \Gamma^{-1}=\dot{\Gamma} \Gamma^{-1}(1-\gamma)
$$

and

$$
\dot{\Gamma} \Gamma^{-1} \gamma=(1-\gamma) \dot{\Gamma} \Gamma^{-1}
$$

Finally,

$$
\begin{aligned}
\hat{\gamma} \Gamma & =\left(\sum_{i}^{n} \dot{\gamma}_{i} \gamma_{i}\right) \Gamma \\
& =\sum_{1}^{n}\left[\dot{\Gamma} \Gamma^{-1}, \gamma_{i}\right] \gamma_{i} \Gamma \quad(\text { by } 4.7) \\
& =\sum_{1}^{n}\left\{\dot{\Gamma} \Gamma^{-1} \gamma_{i}-\gamma_{i} \dot{\Gamma} \Gamma^{-1} \gamma_{i}\right\} \Gamma
\end{aligned}
$$


This last expression coincides with $\dot{\Gamma}$ because $\gamma_{i} \dot{\Gamma} \Gamma^{-1}=\dot{\Gamma} \Gamma^{-1}\left(1-\gamma_{i}\right)$ by (4.9) and therefore $\gamma_{i} \dot{\Gamma} \Gamma^{-1} \gamma_{i}=\dot{\Gamma} \Gamma^{-1}\left(1-\gamma_{i}\right) \gamma_{i}=0$. This proves the theorem.

4.11. REMARK. In general, if $\gamma:[\alpha, \beta] \rightarrow Q_{n}$ is a curve with origin $q^{\prime}=g_{0} q g_{0}^{-1}$ then $\Gamma$ is the horizontal lifting with origin $g_{0}$ if and only if it is the solution of the problem $\dot{\Gamma}=\hat{\gamma} \Gamma, \Gamma(\alpha)=g_{0}$.

We compute next the 1-form, the 2-form and the curvature form of the connection.

We recall that the 1 -form $\theta$ assigns to each $X \in(T G)_{g}$ the horizontal component of $g^{-1} X \in(T G)_{1}=\mathscr{L}$, the Lie algebra of $H$. More explicitly,

$$
\theta_{g} X=v_{1}\left(g^{-1} X\right)=g^{-1} v_{g}(X)=\sum_{i=1}^{n} q_{i} g^{-1} X q_{i}
$$

The 2-form $d \theta$ of the connection is defined by

$$
d \theta(X, Y)=\frac{1}{2}\{X \cdot \theta Y-Y \cdot \theta X-\theta([X, Y])\},
$$

where $X, Y \in(T G)_{g}$, [ , ] denotes the Lie bracket and $Z \cdot W$ denotes the derivative of $W$ in the direction of $Z$, i.e. $W$ is extended to a vector field on a neighborhood of $g$ and given a curve $\delta:(-\varepsilon, \varepsilon) \rightarrow G$ such that $\delta(0)=g$ and $\dot{\delta}(0)=Z$,

$$
Z \cdot W=\frac{d}{d t_{t=0}} W(\delta(t)) .
$$

Although the notation is the same, the Lie bracket should not be confused with the commutator bracket of the algebra.

From the computations

$$
\begin{aligned}
X \cdot \theta Y & =X \cdot\left(\sum_{i=1}^{n} q_{i} g^{-1} Y q_{i}\right) \\
& =-\sum_{i=1}^{n} q_{i} g^{-1} X g^{-1} Y q_{i}+\sum_{i=1}^{n} q_{i} g^{-1} X \cdot Y q_{i}, \\
Y \cdot \theta X & =-\sum_{i=1}^{n} q_{i} g^{-1} Y g^{-1} X q_{i}+\sum_{i=1}^{n} q_{l} g^{-1} Y \cdot X q_{l},
\end{aligned}
$$

and

$$
\theta([X, Y])=\sum_{i=1}^{n} q_{i} g^{-1}[X, Y] q_{i}
$$


we get

$$
\begin{aligned}
d \theta(X, Y) & =\frac{1}{2} \sum_{i=1}^{n} q_{i}\left[g^{-1} Y, g^{-1} X\right] q_{i} \\
& =\left(-\frac{1}{2}\right) \sum_{i=1}^{n} q_{i}\left[g^{-1} X, g^{-1} Y\right] q_{i}
\end{aligned}
$$

The horizontal differential of $\theta$, also called the curvature form of the connection is $\Omega(X, Y)=d \theta\left(h_{g} X, h_{g} Y\right)$ for $[X, Y] \in(T G)_{g}$. Explicitly

$$
\begin{aligned}
& \Omega(X, Y)=\left(-\frac{1}{2}\right) \sum_{i=1}^{n} q_{i}\left[g^{-1} h_{g} X, g^{-1} h_{g} Y\right] q_{i} \\
& \quad=\left(-\frac{1}{2}\right) \sum_{i=1}^{n} q_{i}\left[\sum_{k \neq l} q_{k} g^{-1} X q_{l}, \sum_{r \neq s} q_{r} g^{-1} Y q_{s}\right] q_{i} \\
& \quad=\left(-\frac{1}{2}\right) \sum_{i=1}^{n} q_{i} g^{-1}\left\{X\left(1-q_{i}\right) g^{-1} Y-Y\left(1-q_{i}\right) g^{-1} X\right\} q_{i} \\
& =\left(-\frac{1}{2}\right) \sum_{i=1}^{n} q_{i} g^{-1}\left\{X \bar{q}_{i} g^{-1} Y-Y \bar{q}_{i} g^{-1} X\right\} q_{i}, \\
& \quad=\left(-\frac{1}{2}\right) \sum_{i=1}^{n} q_{i} g^{-1}\left(X g^{-1} Y-Y g^{-1} X-X q_{i} g^{-1} Y+Y q_{i} g^{-1} X\right) q_{i} .
\end{aligned}
$$

The structure equation $\Omega(X, Y)=d \theta(X, Y)+\left(\frac{1}{2}\right)[\theta X, \theta Y]$ is thus trivially satisfied.

5. Calculations on the tangent bundle, geodesics. Consider $q \in Q_{n}$ fixed and let $A_{1}=\left\{X \in A: q_{i} X q_{i}=0, i=1, \ldots, n\right\}$ (in $\S 4$ we called it $\left.H_{1}\right)$. It is clear that $H=\left\{g \in G: g q_{i}=q_{i} g, i=1, \ldots, n\right\}$ operates at left on $A_{1}$ by $h \cdot X:=h X h^{-1}$.

Thus we define the associated bundle of $\pi: G \rightarrow Q_{n}$ with standard fibre $A_{1}$, denoted by $G \otimes A_{1} \rightarrow Q_{n}$, where $G \otimes A_{1}:=G \times A_{1} / \sim$, $(g, X) \sim\left(g h, h^{-1} X\right)$ for $h \in H$ and the map $G \otimes A_{1} \rightarrow Q_{n}$ is determined by $(g, X) \rightarrow \pi(g)$. It is a general fact that this vector bundle is isomorphic to the tangent bundle $T Q_{n}$, by means of $(g, X) \rightarrow\left(\pi(g), g X g^{-1}\right) \in\left(T Q_{n}\right)_{\pi(g)}$. Given a curve $\gamma:[\alpha, \beta] \rightarrow$ 
$Q_{n}$ the parallel displacement of the fibre $\left(T Q_{n}\right)_{\gamma(\alpha)}$ along $\gamma$ from $\alpha$ to $t \in[\alpha, \beta]$ is defined by $\tau_{\alpha}^{t}:\left(T Q_{n}\right)_{\gamma(\alpha)} \rightarrow\left(T Q_{n}\right)_{\gamma(t)}, \tau_{\alpha}^{t}(Z)=$ $\Gamma(t) Z \Gamma(t)^{-1}$, where $\Gamma$ is the horizontal lifting of $\gamma$ with origin $\Gamma(\alpha)=1$.

Given $X \in\left(T Q_{n}\right)_{q}$ and a vector field $Z$ defined near $q$ the covariant derivative $D_{X} Z$ is $D_{X} Z:=X \cdot Z+[Z, \widetilde{X}]$, where

$$
\tilde{X}=\sum_{i=1}^{n} X_{i} q_{i} \text { and } X \cdot Z=\frac{d}{d t_{t=0}} Z(\delta(t))
$$

for a curve $\delta:(-\varepsilon, \varepsilon) \rightarrow Q_{n}$ such that $\delta(0)=q$ and $\dot{\delta}(0)=X$.

5.1. Proposition. For every curve $a:[\alpha, \beta] \rightarrow A^{n}$ the element $D a / d t=\dot{a}+[a, \hat{\gamma}]$ is well defined and has the following properties:

(a) if $\gamma_{i} a \gamma_{i}=0$ for all $i=1, \ldots, n$ then $\gamma_{i}(D a / d t) \gamma_{i}=0$ for all $i=1, \ldots, n$ (in other words, $D a / d t$ is tangent if $a$ is tangent).

(b) if $\gamma_{i} a \gamma_{k}=0$ for all $i \neq k$ then $\gamma_{i}(D a / d t) \gamma_{k}=0$ for all $i \neq k$ (i.e. $D a / d t$ is normal if a is normal).

Proof. (a) Differentiating $\gamma_{i} a \gamma_{i}=0$ we get

$$
0=\dot{\gamma}_{i} a \gamma_{i}+\gamma_{i} \dot{a} \gamma_{i}+\gamma_{i} a \dot{\gamma}_{i} \text {. }
$$

Multiplying by $\gamma_{i}$ at right and left we have

$$
\gamma_{i} \dot{\gamma}_{i} a \gamma_{i}+\gamma_{i} \dot{a} \gamma_{i}+\gamma_{i} a \dot{\gamma}_{i} \gamma_{i}=0 \text {. }
$$

On the other hand

$$
\begin{aligned}
\gamma_{i} \frac{D a}{d t} \gamma_{i} & =\gamma_{i} \dot{a} \gamma_{i}+\gamma_{i}[a, \hat{\gamma}] \gamma_{i} \\
& =\gamma_{i} \dot{a} \gamma_{i}+\gamma_{i}\left(a \sum \dot{\gamma}_{k} \gamma_{k}-\sum \dot{\gamma}_{k} \gamma_{k} a\right) \gamma_{i} \\
& =\gamma_{i} \dot{a} \gamma_{i}+\gamma_{i} a \dot{\gamma}_{i} \gamma_{i}-\gamma_{i} \sum \dot{\gamma}_{k} \gamma_{k} a \gamma_{i}
\end{aligned}
$$

and $\gamma_{i} \sum_{k} \dot{\gamma}_{k} \gamma_{k}=\gamma_{i} \sum_{k}\left(1-\gamma_{k}\right) \dot{\gamma}_{k}$ because $\dot{\gamma}_{k}=\dot{\gamma}_{k} \gamma_{k}+\gamma_{k} \dot{\gamma}_{k}$ (differentiate $\gamma_{k}^{2}=\gamma_{k}$ ); thus

$$
\gamma_{i} \sum_{k} \dot{\gamma}_{k} \gamma_{k}=\gamma_{i} \sum_{k} \dot{\gamma}_{k}-\gamma_{i} \sum \gamma_{k} \dot{\gamma}_{k}=-\gamma_{i} \dot{\gamma}_{i},
$$

because $\sum_{k} \dot{\gamma}_{k}=0$ and $\gamma_{i} \gamma_{k}=0$ if $i \neq k$.

This shows that

$$
\gamma_{i} \frac{D a}{d t} \gamma_{i}=\gamma_{i} \dot{a} \gamma_{i}+\gamma_{i} a \dot{\gamma}_{i} \gamma_{i}+\gamma_{i} \dot{\gamma}_{i} a \gamma_{i}=0, \quad \text { by (4.2). }
$$

The proof of $(b)$ is similar. 
This shows that for every vector field $Y$ of $Q_{n}$ along $\gamma$, the formula $\mathrm{Da} / d t=\dot{Y}+[Y, \hat{\gamma}]$ defines another vector field of $Q_{n}$, the covariant derivative of $Y$.

The torsion of the connection, defined by $T(X, Y)=D_{X} Y-D_{Y} X-$ $[X, Y]$ in general, turns out to be in our case

$$
T(X, Y)=[Y, \tilde{X}]-[X, \tilde{Y}],
$$

where $X, Y \in\left(T Q_{n}\right)_{g}$ and $\widetilde{X}=\sum_{i=1}^{n} X_{i} q_{i}, \widetilde{Y}=\sum_{i=1}^{n} Y_{i} q_{i}$.

5.4. REMARK. For $n=2$ the connection is symmetric, in the sense that its torsion is zero everywhere: in fact, for $n=2$ we have $X_{1}+X_{2}=0, Y_{1}+Y_{2}=0, q_{1}+q_{2}=1, q_{i} X_{i}=X_{i}\left(1-q_{i}\right), q_{i} X_{j}=$ $-X_{i} q_{j}$.

These equalities, when replaced in (4.3), prove the assertion. However, for $n>3$ this is no longer true.

The curvature of the connection, expressed by $R(X, Y) Z=$ $D_{X}\left(D_{Y} Z\right)-D_{Y}\left(D_{X} Z\right)-D_{[X, Y]} Z$ for $X, Y, Z \in\left(T Q_{n}\right)_{q}$, is given, in our case, by

$$
R(X, Y) Z=\left[\sum_{i=1}^{n}\left[X_{i}, Y_{i}\right] q_{i}, Z\right]
$$

or, abbreviating

$$
R(X, Y) Z=\left[[X, Y]^{\sim}, Z\right] .
$$

We study now the geodesic curves of the connection, that is, the curves $\gamma:[\alpha, \beta] \rightarrow Q_{n}$ such that $D \dot{\gamma} / d t=0$. It is a well-known fact that this condition is equivalent to $\tau_{\alpha}^{t}(\dot{\gamma}(\alpha))=\dot{\gamma}(t),(t \in[\alpha, \beta])$. The equation defining the geodesic curves can be written as

$$
\ddot{\gamma}_{k}+\left[\dot{\gamma}_{k}, \hat{\gamma}\right]=0, \quad k=1, \ldots, n \text {. }
$$

Using the commutation rules obtained from $\sum \gamma_{i}=1, \gamma_{i}^{2}=\gamma_{i}$ and $\gamma_{i} \gamma_{k}=0$ for $i \neq k$, we get

(i) $\dot{\gamma}_{i} \gamma_{i}=\left(1-\gamma_{i}\right) \dot{\gamma}_{i}(i=1, \ldots, n)$;

(ii) $\dot{\gamma}_{i} \gamma_{k}+\gamma_{i} \dot{\gamma}_{k}=0 \quad(i \neq k)$;

(iii) $\sum_{i}^{n} \dot{\gamma}_{k}=0$;

(iv) $\gamma_{i} \dot{\gamma}_{i}^{2}=\dot{\gamma}_{i}^{2} \gamma_{i} \quad(i=1, \ldots, n)$;

(v) $\gamma_{i} \dot{\gamma}_{i} \gamma_{i}=0(i=1, \ldots, n)$.

These equalities imply that $(5.7)$ is equivalent to

(5.8) $\ddot{\gamma}_{k}+\gamma_{k}\left(\sum_{1}^{n} \dot{\gamma}_{i}^{2}\right)+\left(\sum_{1}^{n} \dot{\gamma}_{i}^{2}\right) \gamma_{k}-2 \dot{\gamma}_{k}^{2}=0, \quad(k=1, \ldots, n)$. 
It is easy to exhibit all the solutions of (5.8) which satisfy $\gamma(t) \in Q_{n}$ for all $t$. In fact, for $q \in Q_{n}, X \in\left(T Q_{n}\right)_{q}, \gamma(t)=e^{t \widetilde{X}} q e^{-t \widetilde{X}} \quad(t \in R)$, satisfies (5.8) and all the solutions of (5.8) with the additional condition $\gamma(t) \in Q_{n}$, have this form. The connection is also complete, in the sense that its geodesics are defined for all $t \in R$, and the exponential map of the connection is given by

$$
\operatorname{Exp}_{q}:\left(T Q_{n}\right)_{q} \rightarrow Q_{n}, \quad \operatorname{Exp}_{q}(X)=e^{\widetilde{X}} q e^{-\widetilde{X}} .
$$

Properties of minimality of length of geodesics are studied in a forthcoming paper ([CPR2]).

\section{REFERENCES}

[AS] E. Andruchow and D. Stojanoff, Nilpotent operator and systems of projectors, J. Operator Theory, (to appear).

[AFHV] C. Apostol, L. A. Fialkow, D. A. Herrero, and D. Voiculescu, Approximation of Hilbert Space Operators, Vol. II, Pitman, Boston, 1984.

[Au] B. Aupetit, Projections in real Banach algebras, Bull. London Math. Soc., 13 (1981), 412-414.

[Ba] B. A. Barnes, Algebraic elements of a Banach algebra modulo an ideal, Pacific J. Math., 117 (1985), 219-231.

[CPR1] G. Corach, H. Porta, and L. Recht, Two $C^{*}$-algebra inequalities, "Analysis in Urbana", Proceedings of the special year in Modern Analysis at the University of Illinois, Cambridge University Press, London Math. Soc. Lecture Notes \#138, 1989.

[CPR2] _ The geometry of spaces of projectors in $C^{*}$-algebras, to appear in Advances in Math.

[CH] R. Curto, and D. A. Herrero, On closures of joint similarity orbits, Integral Eq. Operator Theory, 8 (1985), 489-556.

[DK1] Ju. L. Daleckii, and S. G. Krein, Properties of operators depending on a parameter, Dopovidi. Akad. Nauk, Ukrain. RSR, 6 (1950), 433-436 (Ukrainian).

[DK2] - Stability of solutions of differential equations in Banach space, Transl. Math. Monographs, vol. 43, Amer. Math. Soc., Providence, R.I., 1974.

[Ga] L. M. Garrido, Generalized adiabatic invariance, J. Math. Phys., 5 (1964), 355-362.

[Gr] B. Gramsch, Relative Inversion in der Störungstheorie von Operatoren und

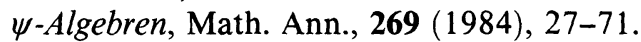

[GS] L. M. Garrido, and F. J. Sancho, Degree of approximate validity of the adiabatic invariance in quantum mechanics, Physica, 28 (1962), 553-560.

[HK] H. Haahti, et S. Kinnunen, Deux connexions linéaires sur des hypersurfaces d'un espace de Banach ou de Minkowski, C. R. Acad. Sci. Paris, t. 305, Ser I (1987), 685-688.

[Ja] N. Jacobson, Structure of rings, Amer. Math. Soc. Coll. Publ., Vol. 36, Providence, R. I., 1956.

[Ka1] T. Kato, Perturbation Theory for Linear Operators, 2nd edition, SpringerVerlag, Berlin, 1984. 
[Ka2] T. Kato On the adiabatic theorem of quantum mechanics, J. Phys. Soc. Japan, 5 (1950), 435-439.

[Ki] S. Kinnunen, Linear connections on hypersurfaces of Banach spaces, Ann. Acad. Sci. Fenn., Ser A, Dissertationes 65, 1987.

[KN] S. Kobayashi, and K. Nomizu, Foundations of Differential Geometry, Vol. I, Interscience, New York, 1963.

[Ko] Z. V. Kovarik, Similarity and interpolation between projectors, Acta Sci. Math., 39 (1977), 341-351.

[La] S. Lang, Differentiable Manifolds, Addison-Wesley, Reading, Mass., 1972.

[Mi] E. Michael, Convex structures and continuous selections, Canad. J. Math., 4 (1959), 556-575.

[PR1] H. Porta, and L. Recht, Spaces of projections in Banach algebras, Acta Cient. Venezolana, 39 (1987), 408-426; it has appeared in preprint form: Report No. 22, Dep. Mat. Comp., Universidad Simón Bolivar, Caracas, 1977.

[PR2] _ Minimality of geodesics in Grassman manifolds, Proc. Amer. Math. Soc., 100, No. 3 (1987).

[Ra] I. Raeburn, The relationship between a commutative Banach algebra and its maximal ideal space, J. Funct. Anal., 25 (1977), 366-390.

[Ri] C. E. Rickart, General Theory of Banach Algebras, Van Nostrand, New York, 1960.

[So] A. Soltysiak, On Banach algebras with closed set of algebraic elements, Ann. Soc. Math. Pol., Ser. I, Commentat. Math., 20 (1978), 479-484.

[Sp] E. H. Spanier, Algebraic Topology, McGraw-Hill, New York, 1966.

[St] N. Steenrod, The Topology of Fiber Bundles, Princeton Univ. Press, Princeton, N. J., 1951.

[Ze] J. Zemánek, Idempotents in Banach algebras, Bull. London Math. Soc., 11 (1979), 177-183.

Received May 26, 1987 and in revised form April 15, 1989.

InSTITUto ARgentino de Matemática

VIAMONTE 1636

1055 Buenos Aires, Argentina

UNIVERSITY OF ILLINOIS

URBANA, IL 61801, U.S.A.

AND

UNiversidad Simón Bolf́var

Caracas, Venezuela 


\section{PACIFIC JOURNAL OF MATHEMATICS EDITORS}

\author{
V. S. VARADARAJAN \\ (Managing Editor) \\ University of California \\ Los Angeles, CA 90024-1555-05 \\ Herbert Clemens \\ University of Utah \\ Salt Lake City, UT 84112 \\ ThOMAs ENRIGHT \\ University of California, San Diego \\ La Jolla, CA 92093
}

R. FINN

Stanford University

Stanford, CA 94305

HeRmanN FlaschKa

University of Arizona

Tucson, AZ 85721

VAUGHAN F. R. JoNES

University of California

Berkeley, CA 94720

SteVen KerCKhofF

Stanford University

Stanford, CA 94305
ROBION KIRBY

University of California

Berkeley, CA 94720

C. C. Moore

University of California

Berkeley, CA 94720

HAROLD STARK

University of California, San Diego

La Jolla, CA 92093

\section{ASSOCIATE EDITORS}
R. ARENS
E. F. BECKENBACH
B. H. NeumanN
F. Wolf
K. YoshidA
(1906-1982)
(1904-1989)

\section{SUPPORTING INSTITUTIONS}

UNIVERSITY OF ARIZONA
UNIVERSITY OF BRITISH COLUMBIA
CALIFORNIA INSTITUTE OF TECHNOLOGY
UNIVERSITY OF CALIFORNIA
MONTANA STATE UNIVERSITY
UNIVERSITY OF NEVADA, RENO
NEW MEXICO STATE UNIVERSITY
OREGON STATE UNIVERSITY
UNIVERSITY OF ARIZONA
UNIVERSITY OF BRITISH COLUMBIA
UNIVERSITY OF CALIFORNIA
MONTANA STATE UNIVERSITY
NEW MEXICO STATE UNIVERSITY

\author{
UNIVERSITY OF OREGON \\ UNIVERSITY OF SOUTHERN CALIFORNIA \\ STANFORD UNIVERSITY \\ UNIVERSITY OF HAWAII \\ UNIVERSITY OF TOKYO \\ UNIVERSITY OF UTAH \\ WASHINGTON STATE UNIVERSITY \\ UNIVERSITY OF WASHINGTON
}

The Supporting Institutions listed above contribute to the cost of publication of this Journal, but they are not owners or publishers and have no responsibility for its content or policies.

Mathematical papers intended for publication in the Pacific Journal of Mathematics should be in typed form or offset-reproduced (not dittoed), double spaced with large margins. Please do not use built up fractions in the text of the manuscript. However, you may use them in the displayed equations. Underline Greek letters in red, German in green, and script in blue. The first paragraph must be capable of being used separately as a synopsis of the entire paper. In particular it should contain no bibliographic references. Please propose a heading for the odd numbered pages of less than 35 characters. Manuscripts, in triplicate, may be sent to any one of the editors. Please classify according to the 1980 Mathematics Subject Classification (1985 Revision) scheme which can be found in the December index volumes of Mathematical Reviews. Supply name and address of author to whom proofs should be sent. All other communications should be addressed to the managing editor, or Elaine Barth, University of California, Los Angeles, California 90024-1555-05.

There are page-charges associated with articles appearing in the Pacific Journal of Mathematics. These charges are expected to be paid by the author's University, Government Agency or Company. If the author or authors do not have access to such Institutional support these charges are waived. Single authors will receive 50 free reprints; joint authors will receive a total of 100 free reprints. Additional copies may be obtained at cost in multiples of 50 .

The Pacific Journal of Mathematics is issued monthly as of January 1966. Regular subscription rate: $\$ 190.00$ a year (6 Vols., 12 issues). Special rate: $\$ 95.00$ a year to individual members of supporting institutions.

Subscriptions, orders for numbers issued in the last three calendar years, and changes of address should be sent to Pacific Journal of Mathematics, P.O. Box 969, Carmel Valley, CA 93924, U.S.A. Old back numbers obtainable from Kraus Periodicals Co., Route 100, Millwood, NY 10546.

The Pacific Journal of Mathematics at P.O. Box 969, Carmel Valley, CA 93924 (ISSN 0030-8730) is published monthly. Second-class postage paid at Carmel Valley, California 93924, and additional mailing offices. Postmaster: send address changes to Pacific Journal of Mathematics, P.O. Box 969, Carmel Valley, CA 93924.

\section{PUBLISHED BY PACIFIC JOURNAL OF MATHEMATICS, A NON-PROFIT CORPORATION}




\section{Pacific Journal of Mathematics}

\section{Vol. 143, No. $2 \quad$ April, 1990}

Gustavo Corach, Horacio Porta and Lázaro Recht, Differential geometry of systems of projections in Banach algebras ................. 209

Peter Fleischmann and Jens Carsten Jantzen, Simple periodic modules of

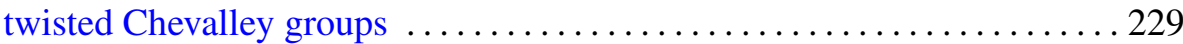

Niels Gronbaek, Amenability of discrete convolution algebras, the commutative case ...................................243

Nguyên H. V. Hung, The mod 2 equivariant cohomology algebras of

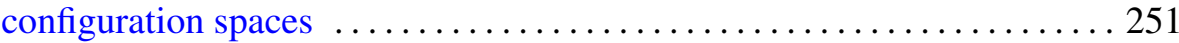

Wojciech Kucharz, Global almost analytic algebraicity of analytic sets . . . 287 John Merrill, A class of consistent anti-Martin's axioms .............. 301

Takafumi Murai, The power 3/2 appearing in the estimate of analytic

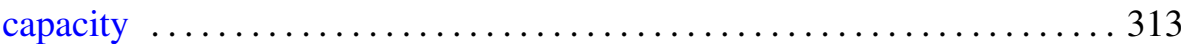

L. Panaitopol and Doru Stefanescu, On the generalized difference

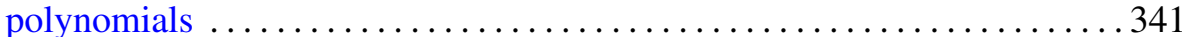

Katsuhiro Shiohama, Takashi Shioya and Minoru Tanaka, Mass of rays

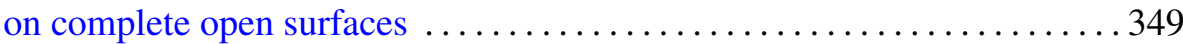

Gerhard Ströhmer, About compressible viscous fluid flow in a bounded

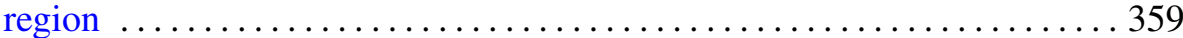

A. Ülger, Arens regularity sometimes implies the RNP 